\title{
Application of chemometric tools for the comparison of volatile profile from raw and roasted regional and foreign almond cultivars (Prunus dulcis)
}

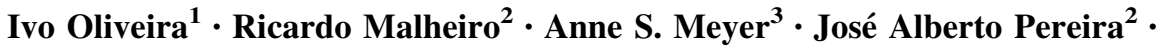 \\ Berta Gonçalves ${ }^{1}$
}

Revised: 18 January 2019/Accepted: 22 May 2019/Published online: 12 June 2019

(C) Association of Food Scientists \& Technologists (India) 2019

\begin{abstract}
In almonds, volatile compounds are major contributors to flavour, being scarce the current knowledge about their volatile profile. Hence, this work intended to characterize the volatile profile, using headspace solidphase microextraction and gas chromatography-mass spectrometry, in raw and roasted almond cultivars (regional cvs. Amendoão, Bonita, Casanova, Molar and Pegarinhos and foreign cvs. Ferragnès and Glorieta). Overall, 35 compounds were identified, with major chemical classes being alcohols and aldehydes. In raw fruits, benzaldehyde and 3-methyl-1-butanol were key compounds, with roasting changing volatile profiles, increasing release of compounds, with predominance of hexanal and benzaldehyde. Cultivars Glorieta and Molar didn't show significant increase in aldehyde content after roasting, which may indicate higher resistance to heat-caused oxidation. The use of linear discriminant analysis and principal components analysis permitted the recognition of patterns in the volatile profiles, that can be useful for cultivars identification. This work allowed the characterization and monitoring changes caused by roasting of volatile components of less studied almond cultivars, identifying some that can withstand
\end{abstract}

Ivo Oliveira

ivo.vaz.oliveira@utad.pt

1 Centre for the Research and Technology of AgroEnvironmental and Biological Sciences - CITAB, Universidade de Trás-os-Montes e Alto Douro, UTAD, Quinta de Prados, 5000-801 Vila Real, Portugal

2 Centro de Investigação de Montanha (CIMO), ESA, Instituto Politécnico de Bragança, Campus de Santa Apolónia, 5300-253 Bragança, Portugal

3 DTU Bioengineering, Department of Biotechnology and Biomedicine, Technical University of Denmark, DTU Building 221, 2800 Kgs, Lyngby, Denmark roasting procedures with reduced formation of compounds associated with off-flavours.

Keywords Almond $\cdot$ Raw $\cdot$ Roasted $\cdot$ Volatiles compounds · Off-flavours · Cultivar discrimination

\section{Introduction}

Considering nut trees, almond (Prunus dulcis (Mill) D. A. Webb) is only surpassed, in production, by cashew and walnut (FAOstat 2018). Almonds are consumed either raw or roasted or as various food applications (Larrauri et al. 2016), and are associated to health-promoting qualities, due to their content in vitamin E, minerals, fatty acid composition, and the presence of minor compounds with antioxidant activity (tocopherols, polyphenols) and cholesterol lowering effects of phytosterols (Sanahuja et al. 2011). However, almond quality is not only linked to its chemical composition, but also to the recognizable flavour that possesses, and that is connected to the aroma component-the volatile composition (Valdés el al. 2015). Unshelled almond are able to maintain their characteristics for about 12 months due to relatively high concentrations of naturally occurring tocopherols (García-Pascual et al. 2003). However, after shelling, but mainly after processing, the changes in the volatile composition of almonds due to oxidation phenomenon can led to onset of unpleasant odour, flavour, colour, and loss of nutrients (McClements and Decker 2010). The major volatile constituents of raw almonds are aldehydes, such as hexanal, nonanal and benzaldehyde (Lee et al. 2014; Mexis et al. 2009; Valdés et al. 2015; Xiao et al. 2014), although ketones, alcohols, alkanes and heterocyclic compounds have been reported (Erten and Cadwallader 2017). For dry roasted almonds 
aldehydes, ketones, alcohols, aromatic hydrocarbons, terpenes, and linear hydrocarbons are those composing the volatile fraction (Erten and Cadwallader 2017). The chemical reactions behind the formation of the majority of volatile compounds in roasted almonds are the Maillard reactions (Agila and Barringer 2012) and results in branched chain aldehydes, alcohols, sulphur-containing and heterocyclic compounds, while straight chain volatiles reflect heat-induced oxidation during roasting (Xiao et al. 2014). Although some works can be found regarding volatile composition of almonds, the large majority was performed in the same cultivars, namely Nonpareil or Butte/Padre, with only few devoted to other important cultivars, like Comuna, Guara or Marcona (Sanahuja et al. 2011; Vazquez-Araujo et al. 2008). Furthermore, cultivars that are currently being increasingly used, like Ferragnès (Berenguer-Navarro et al. 2002) or Glorieta (Romero et al. 2011), or even regional cultivars, that have other traits of interest (Oliveira et al. 2017, 2018) to be used in breeding programs have not been studied, as far as we know, regarding their volatile profile and changes occurring during roasting. Therefore, the present work intends to contribute for the volatile characterization of almond cultivars (regional and foreign ones) in their crude form and after roasting. Were it is hypothesized that some cultivars can have higher resistance to changes caused by roasting, and that volatiles can be a tool to cultivar discrimination. Hence, in this work, we used an HS-SPME GC/MS approach to characterize the volatile profile of several regional almond cultivars and the effect of roasting this profile, comparing them to two foreign cultivars. Due to the importance of this data, chemometric tools, such as Linear Discriminant Analysis (LDA) or Principal Components Analysis (PCA) were applied. In this particular case, chemometrics is important not only to compare cultivars and the technological processes applied, but also to retrieve and interpret important information from the volatile profile patterns. Ultimately, the application of chemometrics, may be useful for varietal discrimination and authentication (as previously verified by Kodad et al. 2011), as well as to attest the application of determined technological processes.

\section{Materials and methods}

\section{Samples}

Almond samples (1 kg), from cultivars commonly produced in Trás-os-Montes, Northeastern Portugal, were obtained directly from producers located in this region. Portuguese cultivars (Amendoão, Molar and Pegarinhos) were collected from the municipality of Murça $\left(41^{\circ} 24^{\prime} 25^{\prime \prime} \mathrm{N}, 7^{\circ} 27^{\prime} 13^{\prime \prime} \mathrm{W}\right)$, and from the municipality of Torre de Moncorvo $\left(41^{\circ} 10^{\prime} 26^{\prime \prime} \mathrm{N} 7^{\circ} 3^{\prime} 0^{\prime \prime} \mathrm{W}\right.$ ) (cvs. Bonita, Casanova, Pegarinhos, and Refêgo). Two foreign cultivars, Ferragnès (French cultivar) and Glorieta (Spanish cultivar), were obtained at a producer from the municipality of Alfândega da Fé $\left(41^{\circ} 20^{\prime} 42^{\prime \prime} \mathrm{N}, 06^{\circ} 58^{\prime} 00^{\prime \prime} \mathrm{W}\right)$. Representative fruits of each cultivar were dehulled (an adequate number to obtain $200 \mathrm{~g}$ of kernels, similar in shape and size, free of visible defects, to be used as raw samples and to be roasted) and roasted, with skin, at $138{ }^{\circ} \mathrm{C}$, for $33 \mathrm{~min}$, to achieve a medium roast. Prior to the introduction of almond samples in the $50 \mathrm{ml}$ vials, raw and roasted samples (10 g per sample, and three samples for each cultivar) were finely crushed using an appliance mill (model A327R1, Moulinex, Spain).

\section{Solid phase micro-extraction (SPME) technique}

For headspace solid-phase microextraction (HS-SPME), a fiber coated with divinylbenzene/carbonex/polydimethylsiloxane (DVB/CAR/PDMS), 50/30 $\mu \mathrm{m}$ was selected based on a preliminary assay conducted alongside two other fibers (CAR/PDMS $75 \mu \mathrm{m}$ and PDMS $100 \mu \mathrm{m})$, all from Supelco (Bellefonte, USA). Selection of the fiber was based on the highest qualitative (number of volatiles extracted) and quantitative data (peak areas) of a sample raw almonds. HS-SPME was conducted according to the methodology applied in other matrices (Malheiro et al. 2013), with some modifications. Almond samples (3 g) were placed in $50 \mathrm{~mL}$ vials and $2 \mathrm{~mL} \mathrm{~g}^{-1}$ of sample of water was added for homogenization purposes. Octanal, 2-methylpyrazine, and 1-hexanol (all from Sigma-Aldrich, St. Louis, MO) internal standards (250 ppm in ethanol) were added at an appropriate amount and the vial was immediately sealed with a polypropylene cap with a silicon septum. The volatiles were released at $40{ }^{\circ} \mathrm{C}$ for $10 \mathrm{~min}$ in an ultrasonic bath. Next, the DVB/CAR/PDMS fiber was exposed for $30 \mathrm{~min}$ at $40{ }^{\circ} \mathrm{C}$ for volatile adsorption, then inserted into the injection port of the $\mathrm{GC}$ system for thermal desorption and reconditioning $\left(10 \mathrm{~min}\right.$ at $\left.220{ }^{\circ} \mathrm{C}\right)$. For each almond cultivar, the HS-SPME analysis was performed in triplicate.

\section{Gas chromatography/mass spectrometry analysis}

The chromatographic conditions were those used by Malheiro et al. (2018). The gas chromatographer used was a Shimadzu GC-2010 Plus equipped with a mass spectrometer Shimadzu GC/MS-QP2010 SE detector. A TRB-5MS $(30 \mathrm{~m} \times 0.25 \mathrm{~mm} \times 0.25 \mu \mathrm{m})$ column (Teknokroma, Spain) was used. The injector was set at $220{ }^{\circ} \mathrm{C}$ and the manual injections were made in splitless mode, with helium (Praxair, Portugal) at a linear velocity of $30 \mathrm{~cm} / \mathrm{s}$ 
and a total flow of $24.4 \mathrm{~mL} / \mathrm{min}$ as mobile phase. The oven temperatures were the following: $40{ }^{\circ} \mathrm{C}(1 \mathrm{~min}) ; 2{ }^{\circ} \mathrm{C} / \mathrm{min}$ until $220^{\circ} \mathrm{C}(30 \mathrm{~min})$. The ionization source was maintained at $250{ }^{\circ} \mathrm{C}$ with ionization energy of $70 \mathrm{eV}$, and with an ionization current of $0.1 \mathrm{kV}$. All mass spectra were acquired by electron ionization in the $\mathrm{m} / \mathrm{z} 35-500$ range. The full scan MS spectra fragments were compared with those obtained from a database (NIST 11) and with those of commercial standards acquired from diverse producers. For qualitative purposes, the areas of the chromatographic peaks were determined by integrating the re-constructed chromatogram from the full scan chromatogram using the ion base $(\mathrm{m} / \mathrm{z}$ intensity $100 \%)$ for each compound (Table 1). For semi-quantification purposes, volatile amounts were calculated by the ratio of each individual base ion peak area to the area of the internal standard base ion peak area and converted to mass equivalents on the basis on the internal standard mass added.

\section{Data analysis}

All data is presented as mean \pm standard deviation, with differences among means determined by analysis of variance (ANOVA), using SPSS (Statistical Package for Social Sciences) software, version 19.0 (IBM Corporation, New York, USA) software. All dependent variables were analysed using a one-way ANOVA, using a data matrix of $23 \times 24$, for raw samples, or $28 \times 24$, for roasted samples (23/28 variables in columns, 24 rows), and comparison of means was performed using Tukey's honestly significant difference multiple comparison test or Dunnett T3 test also depending if equal variances could be assumed or not, with a 5\% significance level.

A linear discriminant analysis (LDA) was used as a supervised learning technique to classify the samples from different cultivars according to their volatile profile. A stepwise technique, using the Wilk's lambda method with the usual probabilities of $\mathrm{F}$ (3.84 to enter and 2.71 to remove), was applied for variable selection. The Wilks' Lambda test was applied to verify which canonical discriminant functions were significant, and to avoid overoptimistic data modulation, the model performance was accessed by a leaving-one-out cross-validation procedure.

The variables identified in the LDA were subsequently used for a Principal components analysis (PCA). This PCA was applied for reducing the number of variables to a smaller number of new derived variables (principal component or factors) that adequately summarize the original information. Moreover, it allows recognizing patterns in the data by plotting them in a multidimensional space, using the new derived variables as dimensions (factor scores).

\section{Results and discussion}

\section{Volatile composition of raw almonds}

The volatile profile of raw and roasted almonds was obtained, and, overall, 35 compounds were tentatively identified (Table 1). Major chemical classes were alcohols and aldehydes, while pyrazines, ketones, terpenes, esters and hydrocarbons were also identified and present in lower extent. The volatile composition of raw almonds was comprised, overall, of 23 compounds, although not all present in each cultivar (Table 2). Total volatile amount ranged from $19.59 \mu \mathrm{g} / \mathrm{g}$ fresh weight $(\mathrm{FW})$, obtained for cv. Ferragnès, to $110.7 \mu \mathrm{g} / \mathrm{g}$ FW for cv. Bonita. Previous studies in almonds have recorded higher number of volatile compounds (Lee et al. 2014; Xiao et al. 2014), while others detected similar (Agila and Barringer 2012; Beck et al. 2011) or lower (Kwak et al. 2015; Mexis et al. 2009; Sanahuja et al. 2011) number of volatiles, which could be related to the methodology or to the cultivar. In half of the cultivars (cvs. Ferragnès, Glorieta, Molar, and Pegarinhos-Moncorvo), alcohols were the major compounds, while, for the remaining cultivars (cvs. Amendoão, Bonita, Casanova and Pegarinhos-Murça), aldehydes represented the larger fraction of the identified volatiles. For the fact that for some cultivars alcohols represent the major fraction of volatiles, it cannot be discarded the effect of the addition of water to the HS-SPME procedure, that is linked to enzymatic reactions occurring in the almond kernel (Kwak et al. 2015). Several compounds were found to be common to all cultivars and included the alcohols benzyl-alcohol, 1-octanol, and phenylethyl-alcohol, as well as the aldehydes hexanal and nonanal. Other major compounds were 3-methyl-1-butanol and benzaldehyde, present in all cultivars studied, being the latter absent in cv. Ferragnès. Several studies indicate aldehydes as the major volatiles, namely benzaldehyde, hexanal or nonanal (Lee et al. 2014; Mexis et al. 2009; Valdés et al. 2015; Xiao et al. 2014). In the studied cultivars, benzaldehyde, a breakdown product of amygdalin (Xiao et al. 2014), was the main volatile only in cvs. Bonita, Casanova, and Pegarinhos-Murça, reporting $79.28 \pm 13.15, \quad 16.93 \pm 8.27$, and $50.07 \pm$ $17.50 \mu \mathrm{g} / \mathrm{g} \mathrm{FW}$, respectively. This compound is a primary element of bitter almonds and is associated to the characteristic bitter taste and aromatic flavour of almonds (Conn 1980). However, this compound, in other cultivars may not be detected (Erten and Cadwallader 2017). Another aldehyde was the major compound in raw almond of $\mathrm{cv}$. Amendoão, namely hexanal $(23.16 \pm 7.62 \mu \mathrm{g} / \mathrm{g} \mathrm{FW})$. This volatile is a product of the auto-oxidation of linolenic acid (Whitfield and Mottram 1992). It is related to the increase of oxidative off-flavors and therefore, its presence may be 
Table 1 Identified volatiles in raw and roasted almonds from regional and foreign cultivars

\begin{tabular}{|c|c|c|c|c|}
\hline Volatile compounds & Class & $\mathrm{LRI}^{\mathrm{a}}$ & LRI Lit. ${ }^{b}$ & $\mathrm{QI}^{\mathrm{c}}$ \\
\hline 3-Methyl-1-butanol & Alcohol & 742 & 740 & 56 \\
\hline 1-Pentanol & Alcohol & 773 & 771 & 42 \\
\hline 3-Penten-2-ol & Alcohol & 780 & 774 & 71 \\
\hline Hexanal & Aldehyde & 799 & 801 & 44 \\
\hline 3-Methyl-1-pentanol & Alcohol & 849 & 843 & 56 \\
\hline 2-Heptanone & Ketone & 891 & 892 & 43 \\
\hline 2-Heptanol & Alcohol & 899 & 905 & 45 \\
\hline Heptanal & Aldehyde & 900 & 899 & 70 \\
\hline 2,5-Dimethyl-pyrazine & Pyrazine & 908 & 911 & 42 \\
\hline$\alpha$-Pinene & Terpene & 936 & 939 & 93 \\
\hline Benzaldehyde & Aldehyde & 959 & 960 & 77 \\
\hline 1-Heptanol & Alcohol & 974 & 966 & 70 \\
\hline 1-Octen-3-ol & Alcohol & 981 & 979 & 57 \\
\hline 3-Octanone & Ketone & 987 & 983 & 43 \\
\hline 6-Methyl-5-hepten-2-one & Ketone & 988 & 985 & 43 \\
\hline 2-Octanone & Ketone & 990 & 991 & 43 \\
\hline 4-Ethylcyclohexanol & Alcohol & 993 & 1003 & 81 \\
\hline Hexyl acetate & Ester & 1015 & 1008 & 43 \\
\hline 3-Ethyl-2-methyl-1,3-hexadiene & Hydrocarbon & 1029 & 1031 & 67 \\
\hline Limonene & Terpene & 1030 & 1029 & 68 \\
\hline Benzyl alcohol & Alcohol & 1034 & 1031 & 43 \\
\hline Benzeneacetaldehyde & Aldehyde & 1044 & 1043 & 91 \\
\hline (E)-2-Octenal & Aldehyde & 1058 & 1054 & 41 \\
\hline 1-Octanol & Alcohol & 1074 & 1068 & 56 \\
\hline 3-Ethyl-2,5-dimethyl-pyrazine & Pyrazine & 1077 & 1082 & 135 \\
\hline Guaiacol & Terpene & 1086 & 1089 & 109 \\
\hline 2-Nonanone & Ketone & 1091 & 1091 & 43 \\
\hline Nonanal & Aldehyde & 1103 & 1100 & 57 \\
\hline Phenylethyl alcohol & Alcohol & 1108 & 1107 & 91 \\
\hline (E)-2-Nonenal & Aldehyde & 1159 & 1161 & 43 \\
\hline Levomenthol & Terpene & 1170 & 1173 & 71 \\
\hline 1-Nonanol & Alcohol & 1172 & 1169 & 56 \\
\hline Methyl salicylate & Ester & 1188 & 1089 & 120 \\
\hline Dodecane & Hydrocarbon & 1202 & 1199 & 57 \\
\hline Decanal & Aldehyde & 1205 & 1201 & 43 \\
\hline
\end{tabular}

${ }^{\mathrm{a}} L R I$ Linear retention index obtained

${ }^{\mathrm{b}}$ LRI Lit Linear retention index reported in literature (Adams 2007)

${ }^{\mathrm{c}}$ Quantification ion associated to some level of oxidation in almonds, mainly after some storage periods (Yang et al. 2013). Inversly it can be associated to green and cut-grass odours in freshly roasted almonds (Erten and Cadwallader 2017). Branchedchain alcohols, common in plant material and result of deamination and decarboxylation of amino acids (Fugelsang and Edwards 2007), represented the major volatiles in the remaining almond cultivars, being 3-methyl-1-butanol the most abundant in cvs. Ferragnès, Glorieta, Molar, and Pegarinhos-Moncorvo. One terpene, limonene, was found in four of the studied cultivars (cvs. Amendoão, Ferragnès, Glorieta, and Molar), while levomenthol was identified in cvs. Molar and Pegarinhos-Moncorvo. The presence of low levels of this type of compounds, or their absence in raw almond samples appears to be usual, as it has been reported elsewhere (Xiao et al. 2014). Guaiacol, a volatile phenol, and methyl salicylate, an ester, were detected only in raw almonds from cv. Molar. The use of a LDA allow the discrimination of the samples (Fig. 1), although those of cvs. Amendoão and Molar were represented together. 
Table 2 Volatile compounds profile and respective concentrations $(\mu \mathrm{g} / \mathrm{g}$ fresh weight \pm standard deviation in brackets; $\mathrm{n}=3$ ) in raw and roasted almonds

\begin{tabular}{|c|c|c|c|c|c|c|c|c|c|c|}
\hline & \multicolumn{2}{|c|}{ Amendoão } & \multicolumn{2}{|l|}{ Bonita } & \multicolumn{2}{|c|}{ Casanova } & \multicolumn{2}{|c|}{ Ferragnès } & \multicolumn{2}{|c|}{ Glorieta } \\
\hline & Raw & Roasted & Raw & Roasted & Raw & Roasted & Raw & Roasted & Raw & Roasted \\
\hline 3-Methyl-1-butanol & $\begin{array}{l}16.23 \\
(7.18)\end{array}$ & $\begin{array}{l}5.48 \\
(3.78)\end{array}$ & $\begin{array}{l}18.43 \\
(0.19)\end{array}$ & $\begin{array}{l}13.79 \\
(4.59)\end{array}$ & $\begin{array}{l}7.79 \\
(0.87)\end{array}$ & $\begin{array}{l}8.40 \\
(0.91)\end{array}$ & $\begin{array}{l}11.82 \\
(2.43)\end{array}$ & $\begin{array}{l}10.97 \\
(3.15)\end{array}$ & $\begin{array}{l}22.15 \\
(4.99)\end{array}$ & $\begin{array}{l}26.26 \\
(16.37)\end{array}$ \\
\hline 1-Pentanol & $\begin{array}{l}0.71 \\
(0.35)\end{array}$ & $\begin{array}{l}1.77 \# \\
(0.30)\end{array}$ & n.d. & n.d. & n.d. & n.d. & n.d. & n.d. & n.d. & n.d. \\
\hline 3-Penten-2-ol & n.d. & n.d. & $\begin{array}{l}1.81 \mathrm{a} \\
(0.32)\end{array}$ & n.d. & n.d. & n.d. & $\begin{array}{l}1.42 \mathrm{ab} \\
(0.47)\end{array}$ & n.d. & n.d. & n.d. \\
\hline Hexanal & $\begin{array}{l}23.16 a \\
(7.62)\end{array}$ & $\begin{array}{l}51.74 \mathrm{~A} \# \\
(3.90)\end{array}$ & $\begin{array}{l}0.69 b \\
(0.53)\end{array}$ & $\begin{array}{l}6.39 \# \mathrm{~B} \\
(0.68)\end{array}$ & $\begin{array}{l}3.94 b \\
(1.31)\end{array}$ & $\begin{array}{l}7.06 \mathrm{~B} \\
(2.42)\end{array}$ & $\begin{array}{l}0.82 \mathrm{~b} \\
(0.80)\end{array}$ & $\begin{array}{l}7.27 \# \mathrm{~B} \\
(0.67)\end{array}$ & $\begin{array}{l}0.27 b \\
(0.07)\end{array}$ & $\begin{array}{l}6.08 \# \mathrm{~B} \\
(1.07)\end{array}$ \\
\hline 3-Methyl-1-pentanol & n.d. & n.d. & n.d. & n.d. & n.d. & n.d. & n.d. & n.d. & $\begin{array}{l}0.23 \\
(0.04)\end{array}$ & n.d. \\
\hline 2-Heptanone & n.d. & $\begin{array}{l}4.81 \mathrm{~A} \\
(2.23)\end{array}$ & n.d. & n.d. & n.d. & n.d. & n.d. & n.d. & n.d. & n.d. \\
\hline 2-Heptanol & n.d. & n.d. & n.d. & n.d. & n.d. & n.d. & n.d. & n.d. & n.d. & n.d. \\
\hline Heptanal & $\begin{array}{l}1.57 \mathrm{a} \\
(0.75)\end{array}$ & $\begin{array}{l}3.80 \mathrm{~A} \\
(2.54)\end{array}$ & n.d. & $\begin{array}{l}0.52 \mathrm{~B} \\
(0.23)\end{array}$ & n.d. & $\begin{array}{l}0.38 \mathrm{~B} \\
(0.12)\end{array}$ & n.d. & $\begin{array}{l}0.31 \mathrm{~B} \\
(0.04)\end{array}$ & $\begin{array}{l}0.21 \mathrm{~b} \\
(0.05)\end{array}$ & $\begin{array}{l}0.43 \mathrm{~B} \\
(0.17)\end{array}$ \\
\hline 2,5-Dimethyl-pyrazine- & n.d. & $\begin{array}{l}0.34 \\
(0.29)\end{array}$ & n.d. & n.d. & n.d. & n.d. & n.d. & n.d. & n.d. & n.d. \\
\hline$\alpha$-Pinene & n.d. & n.d. & n.d. & $\begin{array}{l}7.19 \\
(2.62)\end{array}$ & n.d. & n.d. & n.d. & n.d. & n.d. & $\begin{array}{l}6.25 \\
(3.71)\end{array}$ \\
\hline Benzaldehyde & $\begin{array}{l}2.10 \mathrm{c} \\
(1.32)\end{array}$ & $\begin{array}{l}2.01 \mathrm{~B} \\
(0.81)\end{array}$ & $\begin{array}{l}79.28 \mathrm{a} \\
(13.15)\end{array}$ & $\begin{array}{l}240.97 \# A \\
(37.6)\end{array}$ & $\begin{array}{l}16.93 \mathrm{c} \\
(8.27)\end{array}$ & $\begin{array}{l}\text { 74.23\#B } \\
(31.51)\end{array}$ & n.d. & $\begin{array}{l}6.33 \mathrm{~B} \\
(3.77)\end{array}$ & $\begin{array}{l}0.61 \mathrm{c} \\
(0.43)\end{array}$ & $\begin{array}{l}2.86 \mathrm{~B} \\
(2.49)\end{array}$ \\
\hline 1-Heptanol & $\begin{array}{l}0.81 \mathrm{a} \\
(0.29)\end{array}$ & $\begin{array}{l}1.96 \\
(1.17)\end{array}$ & n.d. & n.d. & n.d. & n.d. & n.d. & $\begin{array}{l}0.26 \\
(0.13)\end{array}$ & $\begin{array}{l}0.22 b \\
(0.09)\end{array}$ & $\begin{array}{l}0.35 \\
(0.21)\end{array}$ \\
\hline 1-Octen-3-ol & $\begin{array}{l}0.32 \\
(0.24)\end{array}$ & $\begin{array}{l}0.53 \\
(0.32)\end{array}$ & n.d. & n.d. & n.d. & n.d. & n.d. & n.d. & $\begin{array}{l}0.05 \\
(0.00)\end{array}$ & n.d. \\
\hline 3-Octanone & n.d. & $\begin{array}{l}0.61 \\
(0.41)\end{array}$ & n.d. & n.d. & n.d. & n.d. & n.d. & n.d. & n.d. & n.d. \\
\hline 6-Methyl-5-hepten-2-one & n.d. & n.d. & n.d. & n.d. & n.d. & n.d. & n.d. & n.d. & n.d. & n.d. \\
\hline 2-Octanone & $\begin{array}{l}1.07 \\
(0.70)\end{array}$ & n.d. & n.d. & n.d. & n.d. & n.d. & n.d. & n.d. & n.d. & n.d. \\
\hline 4-Ethylcyclohexanol & n.d. & n.d. & n.d. & n.d. & n.d. & n.d. & n.d. & n.d. & n.d. & n.d. \\
\hline Hexyl acetate & n.d. & $\begin{array}{l}1.34 \\
(0.94)\end{array}$ & n.d. & n.d. & n.d. & n.d. & n.d. & n.d. & n.d. & n.d. \\
\hline 3-Ethyl-2-methyl-1,3-Hexadiene & n.d. & $\begin{array}{l}1.06 \\
(0.32)\end{array}$ & n.d. & n.d. & n.d. & n.d. & n.d. & n.d. & n.d. & n.d. \\
\hline Limonene & $\begin{array}{l}3.79 \mathrm{a} \\
(0.65)\end{array}$ & n.d. & n.d. & $\begin{array}{l}3.39 \mathrm{~B} \\
(2.59)\end{array}$ & n.d. & $\begin{array}{l}3.90 \mathrm{~B} \\
(2.41)\end{array}$ & $\begin{array}{l}0.75 b \\
(0.11)\end{array}$ & $\begin{array}{l}10.56 \# \mathrm{~A} \\
(2.01)\end{array}$ & $\begin{array}{l}1.47 \mathrm{~b} \\
(0.79)\end{array}$ & $\begin{array}{l}5.25 \mathrm{AB} \\
(2.54)\end{array}$ \\
\hline Benzyl-alcohol & $\begin{array}{l}0.46 \mathrm{~d} \\
(0.35)\end{array}$ & n.d. & $\begin{array}{l}6.74 b \\
(0.97)\end{array}$ & $\begin{array}{l}1.09 \# \mathrm{AB} \\
(0.26)\end{array}$ & $\begin{array}{l}3.79 \mathrm{c} \\
(1.42)\end{array}$ & $\begin{array}{l}0.53 \# \mathrm{~B} \\
(0.15)\end{array}$ & $\begin{array}{l}0.53 \mathrm{~d} \\
(0.11)\end{array}$ & $\begin{array}{l}0.57 \mathrm{~B} \\
(0.39)\end{array}$ & $\begin{array}{l}0.69 d \\
(0.39)\end{array}$ & n.d. \\
\hline Benzeneacetaldehyde & n.d. & $\begin{array}{l}4.94 \mathrm{~A} \\
(3.58)\end{array}$ & n.d. & $\begin{array}{l}0.56 \mathrm{~B} \\
(0.12)\end{array}$ & n.d. & $\begin{array}{l}0.77 \mathrm{~B} \\
(0.25)\end{array}$ & n.d. & $\begin{array}{l}1.12 \mathrm{AB} \\
(0.31)\end{array}$ & n.d. & $\begin{array}{l}1.67 \mathrm{AB} \\
(1.30)\end{array}$ \\
\hline (E)-2-Octenal & n.d. & $\begin{array}{l}0.86 \\
(0.66)\end{array}$ & n.d. & n.d. & n.d. & n.d. & n.d. & n.d. & n.d. & n.d. \\
\hline 1-Octanol & $\begin{array}{l}1.30 \\
(0.31)\end{array}$ & $\begin{array}{l}1.12 \mathrm{~A} \\
(0.61)\end{array}$ & $\begin{array}{l}0.90 \\
(0.32)\end{array}$ & $\begin{array}{l}0.46 \mathrm{~B} \\
(0.04)\end{array}$ & $\begin{array}{l}1.29 \\
(0.58)\end{array}$ & $\begin{array}{l}0.28 \# \mathrm{~B} \\
(0.09)\end{array}$ & $\begin{array}{l}1.37 \\
(0.39)\end{array}$ & $\begin{array}{l}0.29 \# \mathrm{~B} \\
(0.08)\end{array}$ & $\begin{array}{l}1.88 \\
(0.86)\end{array}$ & $\begin{array}{l}0.41 \# \mathrm{~B} \\
(0.10)\end{array}$ \\
\hline
\end{tabular}


Table 2 continued

\begin{tabular}{|c|c|c|c|c|c|c|c|c|c|c|}
\hline & \multicolumn{2}{|c|}{ Amendoão } & \multicolumn{2}{|l|}{ Bonita } & \multicolumn{2}{|c|}{ Casanova } & \multicolumn{2}{|c|}{ Ferragnès } & \multicolumn{2}{|c|}{ Glorieta } \\
\hline & Raw & Roasted & Raw & Roasted & Raw & Roasted & Raw & Roasted & Raw & Roasted \\
\hline 3-Ethyl-2,5-dimethyl-pyrazine & n.d. & $\begin{array}{l}3.78 \\
(2.36)\end{array}$ & n.d. & n.d. & n.d. & n.d. & n.d. & n.d. & n.d. & n.d. \\
\hline Guaiacol & n.d. & n.d. & n.d. & n.d. & n.d. & n.d. & n.d. & n.d. & n.d. & n.d. \\
\hline 2-Nonanone & n.d. & $\begin{array}{l}0.41 \\
(0.31)\end{array}$ & n.d. & n.d. & n.d. & n.d. & n.d. & n.d. & n.d. & n.d. \\
\hline Nonanal & $\begin{array}{l}1.52 \mathrm{a} \\
(0.17)\end{array}$ & $\begin{array}{l}2.82 \\
(1.64)\end{array}$ & $\begin{array}{l}1.29 \mathrm{ab} \\
(0.29)\end{array}$ & $\begin{array}{l}1.35 \\
(0.34)\end{array}$ & $\begin{array}{l}0.62 \mathrm{c} \\
(0.28)\end{array}$ & $\begin{array}{l}1.17 \\
(0.21)\end{array}$ & $\begin{array}{l}0.62 \mathrm{c} \\
(0.22)\end{array}$ & $\begin{array}{l}1.62 \\
(0.60)\end{array}$ & $\begin{array}{l}0.59 \mathrm{c} \\
(0.19)\end{array}$ & $\begin{array}{l}1.60 \# \\
(0.48)\end{array}$ \\
\hline Phenylethyl Alcohol & $\begin{array}{l}0.89 \mathrm{~b} \\
(0.41)\end{array}$ & $\begin{array}{l}0.30 \mathrm{C} \\
(0.19)\end{array}$ & $\begin{array}{l}1.54 b \\
(0.33)\end{array}$ & $\begin{array}{l}0.98 \mathrm{BC} \\
(0.24)\end{array}$ & $\begin{array}{l}1.31 \mathrm{~b} \\
(0.19)\end{array}$ & $\begin{array}{l}0.98 \mathrm{BC} \\
(0.38)\end{array}$ & $\begin{array}{l}2.23 \mathrm{ab} \\
(0.63)\end{array}$ & $\begin{array}{l}1.16 \mathrm{BC} \\
(0.72)\end{array}$ & $\begin{array}{l}1.89 b \\
(0.74)\end{array}$ & $\begin{array}{l}0.69 \mathrm{BC} \\
(0.36)\end{array}$ \\
\hline (E)-2-Nonenal, & n.d. & $\begin{array}{l}0.24 \\
(0.10)\end{array}$ & n.d. & n.d. & n.d. & n.d. & n.d. & n.d. & n.d. & n.d. \\
\hline Levomenthol & n.d. & n.d. & n.d. & n.d. & n.d. & n.d. & n.d. & n.d. & n.d. & n.d. \\
\hline 1-Nonanol & $\begin{array}{l}0.06 \\
(0.04)\end{array}$ & $\begin{array}{l}0.10 \\
(0.05)\end{array}$ & n.d. & n.d. & n.d. & n.d. & n.d. & n.d. & n.d. & n.d. \\
\hline Methyl salicylate & n.d. & n.d. & n.d. & n.d. & n.d. & n.d. & n.d. & n.d. & n.d. & n.d. \\
\hline Dodecane & n.d. & n.d. & n.d. & n.d. & n.d. & n.d. & n.d. & n.d. & n.d. & $\begin{array}{l}0.15 \\
(0.10)\end{array}$ \\
\hline Decanal & n.d. & $\begin{array}{l}0.17 \\
(0.10)\end{array}$ & n.d. & $\begin{array}{l}0.09 \\
(0.02)\end{array}$ & n.d. & $\begin{array}{l}0.08 \\
(0.02)\end{array}$ & n.d. & $\begin{array}{l}0.15 \\
(0.12)\end{array}$ & n.d. & $\begin{array}{l}0.12 \\
(0.02)\end{array}$ \\
\hline \multirow[t]{3}{*}{ Total } & $\begin{array}{l}54.0 \mathrm{bc} \\
(0.49)\end{array}$ & $\begin{array}{l}91.9 \mathrm{~B} \\
(15.4)\end{array}$ & $\begin{array}{l}110.7 \mathrm{a} \\
(13.4)\end{array}$ & $\begin{array}{l}\text { 276.8A\# } \\
(34.6)\end{array}$ & $\begin{array}{l}35.7 \mathrm{~cd} \\
(10.3)\end{array}$ & $\begin{array}{l}97.8 \mathrm{~B} \# \\
(31.0)\end{array}$ & $\begin{array}{l}19.6 \mathrm{~d} \\
(1.74)\end{array}$ & $\begin{array}{l}\text { 40.6B\# } \\
(3.84)\end{array}$ & $\begin{array}{l}30.3 \mathrm{~cd} \\
(3.74)\end{array}$ & $\begin{array}{l}51.9 \mathrm{~B} \# \\
(16.8)\end{array}$ \\
\hline & \multicolumn{2}{|l|}{ Molar } & \multicolumn{3}{|c|}{ Pegarinhos Moncorvo } & \multicolumn{3}{|c|}{$\begin{array}{l}\text { Pegarinhos } \\
\text { Murça }\end{array}$} & \multicolumn{2}{|l|}{$P$ value } \\
\hline & Raw & Roasted & & & Roasted & Raw & Roa & & Raw & Roasted \\
\hline 3-Methyl-1-butanol & $\begin{array}{l}12.20 \\
(6.43)\end{array}$ & $\begin{array}{l}12.69 \\
(3.09)\end{array}$ & & & $\begin{array}{l}13.64 \\
(4.32)\end{array}$ & $\begin{array}{l}13.67 \\
(4.59)\end{array}$ & $\begin{array}{l}11.4 \\
(2.7\end{array}$ & & 0.133 & 0.221 \\
\hline 1-Pentanol & n.d. & n.d. & $\mathrm{n}$. & & n.d. & n.d. & n.d. & & - & - \\
\hline 3-Penten-2-ol & n.d. & n.d. & & & n.d. & n.d. & n.d. & & 0.023 & - \\
\hline Hexanal & $\begin{array}{l}0.57 b \\
(0.30)\end{array}$ & $\begin{array}{l}3.06 \mathrm{~B} \\
(1.86)\end{array}$ & & & $\begin{array}{l}6.05 \# \mathrm{~B} \\
(0.86)\end{array}$ & $\begin{array}{l}1.89 \mathrm{~b} \\
(0.17)\end{array}$ & $\begin{array}{l}4.70 \\
(0.5\end{array}$ & & $<0.001$ & $<0.001$ \\
\hline 3-Methyl-1-pentanol & n.d. & n.d. & & & n.d. & n.d. & n.d. & & 0.864 & - \\
\hline 2-Heptanone & n.d. & $\begin{array}{l}0.45 \mathrm{~B} \\
(0.18)\end{array}$ & $\mathrm{n}$. & & n.d. & n.d. & n.d. & & - & 0.028 \\
\hline 2-Heptanol & n.d. & n.d. & $\mathrm{n}$. & & n.d. & $\begin{array}{l}0.68 \\
(0.26)\end{array}$ & n.d. & & - & - \\
\hline Heptanal & n.d. & $\begin{array}{l}0.52 \mathrm{~B} \\
(0.25)\end{array}$ & $\mathrm{n}$. & & $\begin{array}{l}0.42 \mathrm{~B} \\
(0.05)\end{array}$ & n.d. & $\begin{array}{l}0.45 \\
(0.0\end{array}$ & & 0.036 & 0.003 \\
\hline 2,5-Dimethyl-pyrazine- & n.d. & n.d. & $\mathrm{n}$. & & n.d. & n.d. & n.d. & & - & - \\
\hline$\alpha$-Pinene & n.d. & $\begin{array}{l}2.76 \\
(0.60)\end{array}$ & $\mathrm{n}$. & & $\begin{array}{l}3.87 \\
(1.53)\end{array}$ & n.d. & $\begin{array}{l}4.64 \\
(1.4\end{array}$ & & - & 0.188 \\
\hline Benzaldehyde & $\begin{array}{l}1.18 \mathrm{c} \\
(0.67)\end{array}$ & $\begin{array}{l}2.06 \mathrm{~B} \\
(0.70)\end{array}$ & & & $\begin{array}{l}\text { 166.41\#A } \\
(64.37)\end{array}$ & $\begin{array}{l}50.07 \mathrm{~b} \\
(17.50)\end{array}$ & $\begin{array}{l}204 . \\
(37 .\end{array}$ & $\begin{array}{l}9 \# \mathrm{~A} \\
8)\end{array}$ & $<0.001$ & $<0.001$ \\
\hline 1-Heptanol & n.d. & n.d. & $\mathrm{n}$. & & n.d. & n.d. & n.d. & & 0.030 & 0.204 \\
\hline
\end{tabular}


Table 2 continued

\begin{tabular}{|c|c|c|c|c|c|c|c|c|}
\hline & \multicolumn{2}{|l|}{ Molar } & \multicolumn{2}{|c|}{ Pegarinhos Moncorvo } & \multicolumn{2}{|c|}{$\begin{array}{l}\text { Pegarinhos } \\
\text { Murça }\end{array}$} & \multicolumn{2}{|l|}{$P$ value } \\
\hline & Raw & Roasted & Raw & Roasted & Raw & Roasted & Raw & Roasted \\
\hline 1-Octen-3-ol & n.d. & $\begin{array}{l}0.19 \\
(0.02)\end{array}$ & n.d. & n.d. & n.d. & n.d. & 0.123 & 0.208 \\
\hline 3-Octanone & n.d. & n.d. & n.d. & n.d. & n.d. & n.d. & - & - \\
\hline 6-Methyl-5-hepten-2-one & n.d. & $\begin{array}{l}0.09 \\
(0.03)\end{array}$ & n.d. & n.d. & n.d. & n.d. & - & - \\
\hline 2-Octanone & n.d. & n.d. & n.d. & n.d. & n.d. & n.d. & - & - \\
\hline 4-Ethylcyclohexanol & n.d. & $\begin{array}{l}0.18 \\
(0.16)\end{array}$ & n.d. & n.d. & n.d. & n.d. & - & - \\
\hline Hexyl acetate & n.d. & n.d. & n.d. & n.d. & n.d. & n.d. & - & - \\
\hline 3-Ethyl-2-methyl-1,3-Hexadiene & n.d. & n.d. & n.d. & n.d. & n.d. & n.d. & - & - \\
\hline Limonene & $\begin{array}{l}0.74 b \\
(0.29)\end{array}$ & $\begin{array}{l}1.87 \# B \\
(0.62)\end{array}$ & n.d. & $\begin{array}{l}3.45 \mathrm{~B} \\
(1.42)\end{array}$ & n.d. & $\begin{array}{l}4.21 \mathrm{~B} \\
(1.31)\end{array}$ & $<0.001$ & 0.003 \\
\hline Benzyl-alcohol & $\begin{array}{l}0.42 \mathrm{~d} \\
(0.02)\end{array}$ & n.d. & $\begin{array}{l}9.23 \mathrm{a} \\
(1.19)\end{array}$ & $\begin{array}{l}1.42 \# \mathrm{~A} \\
(0.16)\end{array}$ & $\begin{array}{l}6.93 b \\
(0.46)\end{array}$ & $\begin{array}{l}0.71 \# \mathrm{~B} \\
(0.23)\end{array}$ & $<0.001$ & 0.007 \\
\hline Benzeneacetaldehyde & n.d. & $\begin{array}{l}1.71 \mathrm{AB} \\
(0.86)\end{array}$ & n.d. & $\begin{array}{l}2.38 \mathrm{AB} \\
(0.44)\end{array}$ & n.d. & $\begin{array}{l}2.13 \mathrm{AB} \\
(0.75)\end{array}$ & - & 0.041 \\
\hline (E)-2-Octenal & n.d. & n.d. & n.d. & n.d. & n.d. & n.d. & - & - \\
\hline 1-Octanol & $\begin{array}{l}1.08 \\
(0.29)\end{array}$ & $\begin{array}{l}0.33 \# \mathrm{~B} \\
(0.08)\end{array}$ & $\begin{array}{l}1.49 \\
(0.19)\end{array}$ & $\begin{array}{l}0.44 \# \mathrm{~B} \\
(0.11)\end{array}$ & $\begin{array}{l}1.13 \\
(0.72)\end{array}$ & $\begin{array}{l}0.33 \mathrm{~B} \\
(0.06)\end{array}$ & 0.457 & 0.007 \\
\hline 3-Ethyl-2,5-dimethyl-pyrazine & n.d. & n.d. & n.d. & n.d. & n.d. & n.d. & - & - \\
\hline Guaiacol & $\begin{array}{l}1.35 \\
(0.37)\end{array}$ & n.d. & n.d. & n.d. & n.d. & n.d. & - & - \\
\hline 2-Nonanone & n.d. & n.d. & n.d. & n.d. & n.d. & n.d. & - & - \\
\hline Nonanal & $\begin{array}{l}0.74 b c \\
(0.07)\end{array}$ & $\begin{array}{l}1.09 \# \\
(0.21)\end{array}$ & $\begin{array}{l}1.67 \mathrm{a} \\
(0.29)\end{array}$ & $\begin{array}{l}1.29 \\
(0.38)\end{array}$ & $\begin{array}{l}0.54 \mathrm{c} \\
(0.12)\end{array}$ & $\begin{array}{l}1.24 \\
(0.46)\end{array}$ & $<0.001$ & 0.130 \\
\hline Phenylethyl Alcohol & $\begin{array}{l}1.48 b \\
(0.57)\end{array}$ & $\begin{array}{l}0.88 \mathrm{BC} \\
(0.14)\end{array}$ & $\begin{array}{l}3.79 a \\
(1.25)\end{array}$ & $\begin{array}{l}3.29 \mathrm{~A} \\
(0.67)\end{array}$ & $\begin{array}{l}2.64 \mathrm{ab} \\
(0.18)\end{array}$ & $\begin{array}{l}1.72 \mathrm{~B} \\
(0.55)\end{array}$ & 0.001 & $<0.001$ \\
\hline (E)-2-Nonenal, & n.d. & n.d. & n.d. & n.d. & n.d. & n.d. & - & - \\
\hline Levomenthol & $\begin{array}{l}1.94 \\
(1.53)\end{array}$ & n.d. & $\begin{array}{l}0.75 \\
(0.21)\end{array}$ & n.d. & n.d. & n.d. & 0.253 & - \\
\hline 1-Nonanol & n.d. & n.d. & n.d. & n.d. & n.d. & n.d. & - & - \\
\hline Methyl salicylate & $\begin{array}{l}0.85 \\
(0.46)\end{array}$ & n.d. & n.d. & n.d. & n.d. & n.d. & - & - \\
\hline Dodecane & n.d. & $\begin{array}{l}0.04 \\
(0.00)\end{array}$ & n.d. & n.d. & n.d. & n.d. & - & $<0.001$ \\
\hline Decanal & n.d. & n.d. & n.d. & $\begin{array}{l}0.13 \\
(0.08)\end{array}$ & n.d. & $\begin{array}{l}0.11 \\
(0.04)\end{array}$ & - & 0.698 \\
\hline Total & $\begin{array}{l}22.6 \mathrm{~d} \\
(6.39)\end{array}$ & $\begin{array}{l}27.9 \mathrm{~B} \\
(2.26)\end{array}$ & $\begin{array}{l}47.4 \mathrm{~cd} \\
(9.06)\end{array}$ & $\begin{array}{l}\text { 202.8A\# } \\
(63.9)\end{array}$ & $\begin{array}{l}77.6 \mathrm{~b} \\
(20.5)\end{array}$ & $\begin{array}{l}\text { 236.4A\# } \\
(34.6)\end{array}$ & $<0.001$ & $<0.001$ \\
\hline
\end{tabular}

Different small letters, for each compound, indicate significant differences among raw samples. Different capital letters, for each compound, indicate significant differences among roasted samples

N.d. not detected

\#indicates significant differences between raw and roasted samples, for the same cultivar 
Fig. 1 Linear discriminant analysis of the volatile compounds from raw almond samples, represented in a plane composed by the two main discriminant functions. The functions explain $66.6 \%$ of the total variance
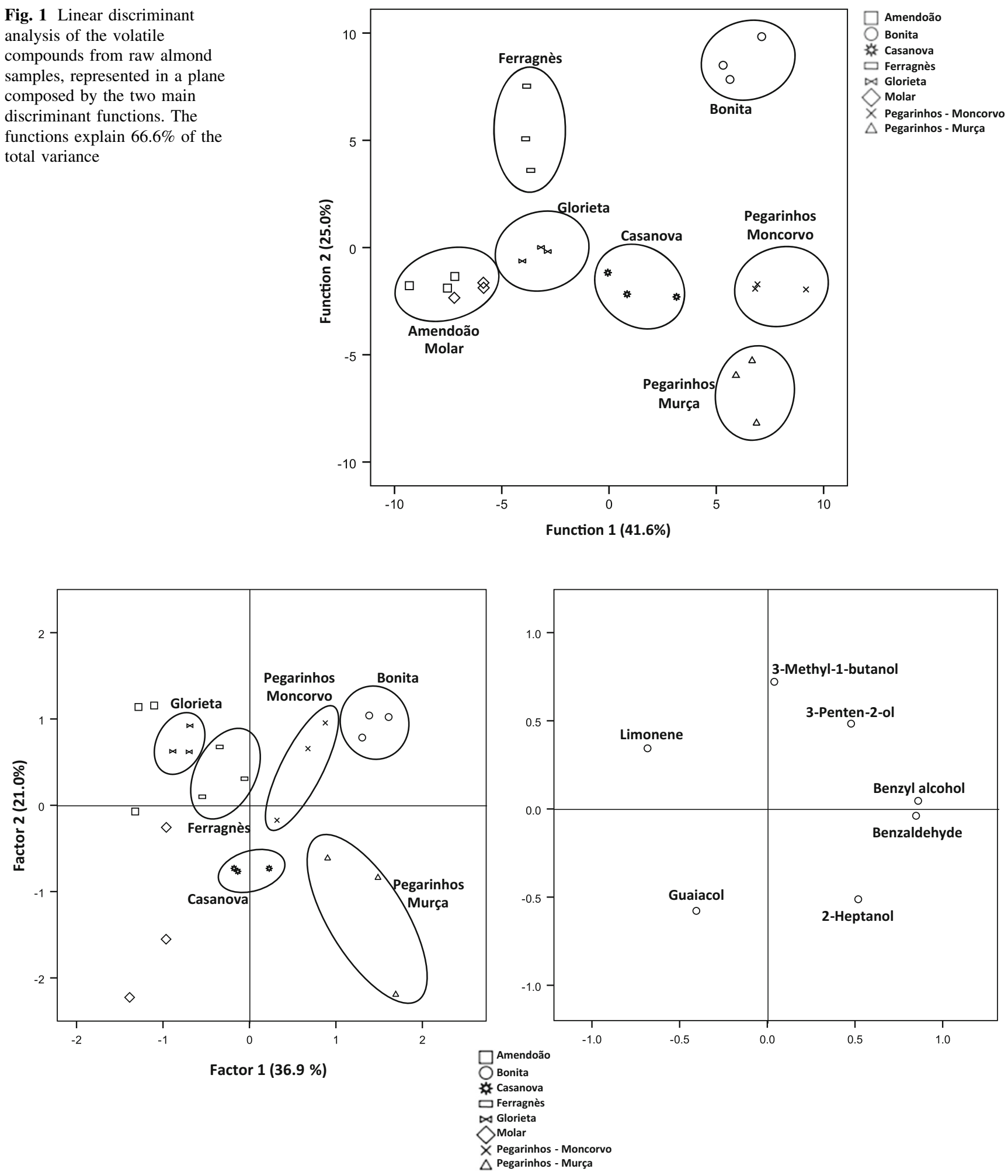

Fig. 2 Principal component analysis of the volatile compounds derived from the LDA analysis of raw almond samples. The factors explain 58\% of the total variance. Missing labels are for cvs. Amendoão (squares) and Molar (diamonds)

The LDA approach makes it possible to identify the significant variables associated to a data set. This analysis reduced the number of the total variables (volatile compounds) to only seven volatiles with statistical significance (benzyl alcohol, 3-penten-2-ol, guaiacol, benzaldehyde, limonene, 2-heptanol, and 3-methyl-1-pentanol), that 
were subsequently used in the PCA. The use of this statistic tool, based on those seven volatiles explained about $58 \%$ of the data (Fig. 2). All samples, with the exception of cvs. Amendoão and Molar, were easily grouped. Samples of cv. Pegarinhos-Murça, located in the positive axis of Factor 1 and negative axis of Factor 2 are clearly influenced by their amount in 2-heptanol, as this compound was only recorded in these samples. Similarly, the content of 3-pentel-2-ol and benzaldehyde, higher in this cultivar, led to the representation of cv. Bonita samples in the positive axis of both factors. Cultivars Ferragnès and Glorieta are influenced by their content in limonene, which resulted in the representation of these samples in the positive axis of Factor 1 and negative axis of factor 2. For the representation of the remaining cultivars, no significant influence of a given chemical group was found.

\section{Changes in volatile compounds during roasting}

Considerable changes in the volatile profile were caused by the roasting of almonds (Table 2). Total volatile amount ranged from $27.95 \mu \mathrm{g} / \mathrm{g} \mathrm{FW}$, obtained for $\mathrm{cv}$. Molar, to $276.76 \mu \mathrm{g} / \mathrm{g}$ FW for cv. Bonita. There was a considerable increase of the aldehydes content in all samples, with a parallel decrease in the alcohol amounts present. However, it should be pointed out that although the relative abundance shifted from alcohols to aldehydes in some cultivars (cvs. Ferragnès and Pegarinhos-Moncorvo), the total amount of volatiles was higher in all roasted samples. Only two cultivars, that appear to be more resistant to changes caused by roasting, cvs. Glorieta and Molar, have alcohols as the main volatile fraction after roasting. In fact, for $\mathrm{cv}$. Glorieta the total amount of alcohols even recorded a slight increase, while, for cv. Molar the reduction was of only $6 \%$, comparing to the reduction recorded for Ferragnès (about less 24\%), while all other cultivars recorded higher losses of alcohols. Glorieta has a percentage of saturated fatty acids higher than several cultivars, including Ferragnes (Yildirim et al. 2016), as does Molar (Silva and Ferreira 1983), that are more stable than unsaturated fatty acids, and therefore less prone to be converted into aldehydes. Furthermore, this low amount of unsaturated fatty acids can also lead to a conversation of some of the present aldehydes into alcohols by alcohol dehydrogenases (Dudareva et al. 2004). For all other almond cultivars, after roasting, aldehydes represent the most abundant compounds. Increases in the total amount of aldehydes occurred in all samples, but more prominently in those of cvs. Ferragnès and Pegarinhos-Moncorvo, in which content of volatile aldehydes increased about 12 times. Samples of cv. Glorieta also recorded a relatively high increase of aldehydes ( 7 times), but, together with samples of cv. Molar (3 times higher content of aldehydes, similar to the remaining cultivars), are those with lower amount of these compounds, for total content and relative abundance. This increase on the amount of aldehydes present is a common pattern (Erten and Cadwallader 2017; Lee et al. 2014; Xiao et al. 2014) and it is linked to the occurrence of several chemical reactions, namely the auto-oxidation or degradation of lipids or the Strecker degradation in the Maillard reaction (Erten and Cadwallader 2017). Hexanal, the major volatile of roasted cv. Amendoão almonds $(51.74 \pm 3.90 \mu \mathrm{g} / \mathrm{g} \mathrm{FW})$, increased significantly with roasting in all cultivars, except for cvs. Casanova and Molar. It is a product from the auto-oxidation of linolenic acid, but is also generated from thermal oxidation of linoleates (Beck et al. 2011). However, those two cultivars have low amounts of linolenic acid (although depending on harvest year and site) when compared to other cultivars (Silva and Ferreira 1983), making them less prone to present hexanal in their volatile composition. Benzaldehyde, an important compound for the flavour of almonds, was the major one in roasted fruits of cvs. Bonita, Casanova, Pegarinhos-Moncorvo, and Pegarinhos-Murça, where a significant increase was recorded. Furthermore, this compound was not present in raw fruits of $\mathrm{cv}$. Ferragnès, appearing in those after roasting. As referred before, benzaldehyde is a product of amygdalin. In turn, amygdalin may be synthesised from phenylalanine that under heat yields benzaldehyde (Durmaz and Gökmen 2010). Therefore, the increase of benzaldehyde in roasted samples is linked both to the use of heat, but also to variations of the content of amygdalin in raw samples, which vary among cultivars (Lee et al. 2013). Roasting of almonds also cause the formation of other volatile aldehydes. Heptanal, that had been detected only in raw cvs. Amendoão and Glorieta, is now present in all roasted samples. As for many other aldehydes, heptanal is formed from the fatty acid decomposition, namely linolenic acid (Mexis et al. 2009) and its increase with roasting of almonds is well recorded (Agila and Barringer 2012; Lee et al. 2014; Xiao et al. 2014). This compound may lead to some flavours, either negative (oily, fatty, heavy) or positive (woody, penetrating, sweet, nutty, fruity) (Krist et al. 2004). Roasting also caused the formation of benzeneacetaldehyde and decanal in all samples. Benzeneacetaldehyde is a compound formed from phenylalanine by action of polyphenol oxidase, and has been found in freshly roasted almonds, with its content decreasing as time passes after processing (Valdés et al. 2015). Its odour descriptors include honey-like scent, harsh, and hawthorn (Krist et al. 2004). By other hand, decanal, described has having aldehyde-like, fruity, soapy odour (Cuevas et al. 2016), is a product of the oxidation of oleic acid (Frankel 1982). Although it has been found in oil of raw almonds (Sanahuja et al. 2011), its occurrence is normally associated to oxidation processes (Valdés et al. 
2015), either by heat (Lee et al. 2014; Xiao et al. 2014). In what concerns aldehydes, it should also be pointed out the presence of $(E)$-2-octenal and $(E)$-2-nonenal in $\mathrm{cv}$. Amendoão, compounds that derive from the oxidation of linoleic acid (Lee et al. 2007). (E)-2-octenal is linked to off-odours (Yang et al. 2013) while (E)-2-nonenal is associated to hay and floral odours (Erten and Cadwallader 2017). Both compounds have been recorded in raw samples, at low concentrations (Agila and Barringer 2012), their content increasing with oxidation caused by different roasting processes (Erten and Cadwallader 2017; Xiao et al. 2014). Alcohols, the major compounds in half of the samples when raw, suffer considerable variation caused by roasting. For cvs. Ferragnès, Glorieta and Molar, the major volatile was 3-methyl-1-butanol, the same as recorded in raw samples. This compound, together with 1-nonanol and phenylethyl alcohol, were the only alcohols that did not show statistically significant variations caused by roasting. This fact has not been previously reported, with other works available showing a significant decrease of 3-methyl-1-butanol with roasting (Xiao et al. 2014) or the formation of 1-nonanol months after the storage of roasted samples but not on freshly roasted ones (Lee et al. 2014). Indeed, previous work show that roasting usually results in the formation of alcohols, along with other compounds (Agila and Barringer 2012; Xiao et al. 2014). However, our results show that some alcohols completely disappeared after samples were roasted, a pattern detected for 3-penten2-ol, 3-methyl-1-pentanol and 2-heptanol. In an inverse situation, 1-pentanol, which is formed by the degradation of linoleic acid hydroperoxides, was present in higher content after roasting of almonds from cv. Amendoão, a pattern similar to the recorded for cvs. Nonpareil and Butte (Franklin et al. 2017; Xiao et al. 2014). The presence of this specific alcohol may be seen as a sign that higher oxidation occurred in this cultivar, and may add fermentative aromas to its odour (Franklin et al. 2017). An effect of the studied cultivar was observed in the content of other alcohols in roasted samples. 1-heptanol, detected in cvs. Amendoão and Glorieta in statistical similar amounts in raw and roasted almonds of those cultivars, was a new compound in roasted samples of cv. Ferragnès. The limited effect of light and medium roasting treatments in this specific compound has been earlier recorded in cv. Butte (Lee et al. 2014; Xiao et al. 2014), although an increase of its amount was found when a more aggressive roasting procedure was used. Although it is a compound that may present positive flavour description (herbal, fragrant, woody), other traits are recognizably negative (pungent, musty, heavy, and oil) (Franklin et al. 2017; Krist et al. 2004). By other hand, 1-octen-3-ol was not affected by roasting on samples of cv. Amendoão, completely disappearing in cv. Glorieta, but being formed in cv. Molar. This compound, formed by thermal decomposition of methyl linoleate hydroperoxide (Min and Smouse 1985), adds an herbaceous aroma (Vazquez-Araujo et al. 2008) to samples. For benzyl-alcohol and 1-octanol, alcohols present in all raw samples, their presence illustrates even more the effect of the cultivar associated to the roasting process. The content of benzyl-alcohol remained unchanged only in cv. Ferragnès, decreasing significantly in cvs. Bonita, Casanova, and Pegarinhos-Moncorvo and Murça and completely disappearing in the remaining samples (Amendoão, Glorieta, and Molar). The roasting process significantly reduced the content of 1-octanol in five of the studied cultivars (cvs. Casanova, Ferragnès, Glorieta, Molar, and Pegarinhos-Moncorvo), although did not cause any changes in samples from cvs. Amendoão, Bonita or Pegarinhos-Murça. The variation of the content of terpenes was affected by the processing of almonds, but, yet again with cultivars presenting different susceptibilities. For $\alpha$-pinene, roasting of almonds led to its formation in five of the samples (cvs. Bonita, Glorieta, Molar, and both samples of Pegarinhos), while for the remaining cultivars this compound was not found, either in raw or roasted samples. Although $\alpha$-pinene, a compound described has having a sharp, stone-pine flavour (Krist et al. 2004) has been reported in raw almonds of cv. Butte (Lee et al. 2014; Xiao et al. 2014) and in an unidentified cultivar (Valdés et al. 2015), not all previous works shows the presence of this compound (Erten and Cadwallader 2017; Kwak et al. 2015). Furthermore, changes in its amounts after processing are only recorded after long term exposure to oxidation procedures (Valdés et al. 2015), while light roasting does not significantly change the presence of $\alpha$-pinene in almonds (Lee et al. 2014; Xiao et al. 2014). For the other terpene detected in some of the samples, limonene, compound with orange-like and fruity odour (Cuevas et al. 2016), that had been found in four of the studied cultivars (cvs. Amendoão, Ferragnès, Glorieta, and Molar), when raw, was present in all except one, in which it completely disappeared (cv. Amendoão). Light roasting has already proved not to cause significant variations on the content of limonene (Lee et al. 2014; Xiao et al. 2014), as recorded in the present work for cv. Glorieta. However, increased levels of roasting can lead to significant reductions of its amounts (Xiao et al. 2014). In contrary, other reports indicate an increase in the total amount of terpenes, including limonene, when almonds are roasted (Agila and Barringer 2012; Yang et al. 2013). Ketones were present is small amounts in roasted almonds, and only in three of the analysed cultivars-cvs. Amendoão, Glorieta, and Molar. Roasting led to the formation of 2-heptanone (samples of cvs. Amendoão and Molar), 3-octanone and 2-nonanone (in samples of cv. Amendoão) and 6-methyl-5-hepten-2-one in samples of cv. Molar. In an inverse way, 2-octanone, that 
was present in raw samples of cv. Amendoão, disappeared after roasting. Formation of ketones with roasting is a known event (Agila and Barringer 2012; Lee et al. 2014; Xiao et al. 2014), as they are widely recognized products of lipid oxidation and have also been looked at as indicators of rancidity (Franklin et al. 2017). In fact, the presence of ketones contributes to off-flavours of nuts (Yang et al. 2013), with described soapy and cheese aroma (Franklin et al. 2017). Two pyrazines (2,5-dimethyl-pyrazine and 3-ethyl-2,5-dimethyl-pyrazine) were only found after roasting and in the samples of cv. Amendão, although they have been reported also in raw almonds (Agila and Barringer 2012; Valdés et al. 2015; Xiao et al. 2014). However, most works point out the positive effect of roasting in the content of these compounds (Agila and Barringer 2012; Erten and Cadwallader 2017; Lee et al. 2014; Xiao et al. 2014), and, in some situations, reporting that they are among the main volatile compounds in roasted almonds (Vazquez-Araujo et al. 2008). These compounds are formed during heating by the Maillard sugar-amine reactions and Strecker degradation and have nutty and roasted aromas (Erten and Cadwallader 2017; Franklin et al. 2017) and improve the roasted aromas of almonds (Yada et al. 2011). Other compounds found in roasted almonds include hexyl acetate and 3-ethyl-2-methyl-1,3-hexadiene, in samples of cv. Amendoão, and dodecane, in samples of cvs. Glorieta and Molar. Using a linear discriminant analysis with data of volatile compounds of roasted samples it was possible to discriminate the samples from $\mathrm{cv}$.
Amendoão and Molar (Fig. 3), while all other cultivars are grouped together. The LDA selected only five volatile compounds (benzaldehyde, hexanal, phenylethyl alcohol, 4-ethylcyclohexanol, and 6-methyl-5-hepten-2-one), that were used to perform a PCA (Fig. 3). That data, explaining $78 \%$ of the total variance of results, allowed a separation of samples in four major groups: cvs. Amendoão, Molar, Casanova, Ferranès, and Glorieta, and finally, cvs. Bonita, Pegarinhos-Moncorvo, and Pegarinhos-Murça. The considerable higher content in hexanal of samples of cv. Amendoão is the main responsible for the separation of this cultivar, while the presence of 4-ethylcyclohexanol and 6-methyl-5-hepten-2-one led to the grouping of cv. Molar samples. As the samples of cvs. Bonita, PegarinhosMoncorvo and Murça presented high content of benzaldehyde, they were grouped together, while the remaining samples (cvs. Casanova, Ferranès, and Glorieta) are represented jointly, as none of the compounds exerts significant influence. The use of LDA and PCA allowed, to some extent, the discrimination of almond cultivars based on few but important volatile compounds that appear to be characteristic of each sample (Fig. 4).

\section{Conclusion}

This work allowed the comparison of volatile profiles of regional and foreign almond cultivars, obtained from raw and roasted fruits. In raw fruits, alcohols were the major
Fig. 3 Linear discriminant analysis of the volatile compounds from roasted almond samples, represented in a plane composed by the two main discriminant functions. The functions explain $98.9 \%$ of the total variance

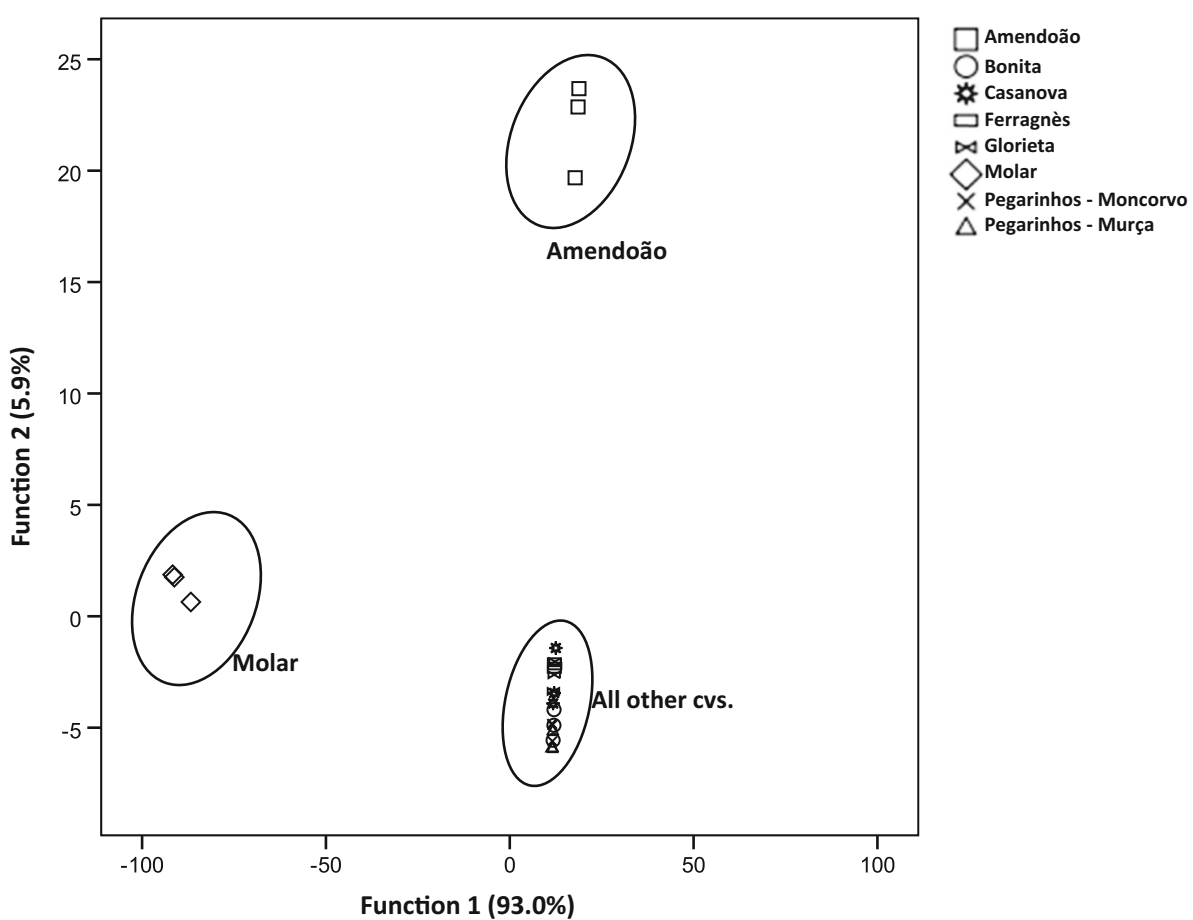




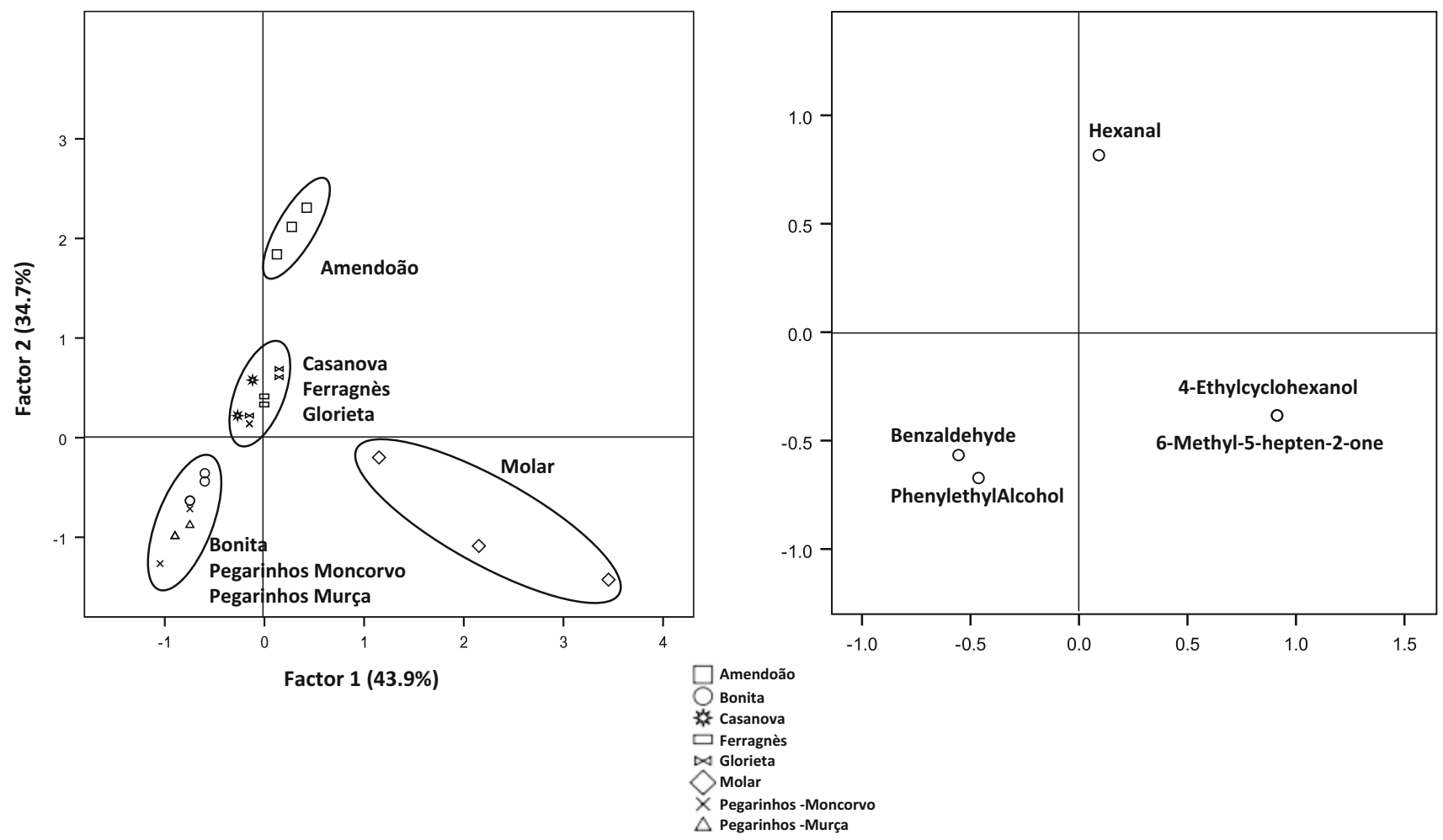

Fig. 4 Principal component analysis of the volatile compounds derived from the LDA analysis of roasted almond samples. The factors explain $78.6 \%$ of the total variance

compounds in half of the cultivars (cvs. Ferragnès, Glorieta, Molar, and Pegarinhos-Moncorvo), while, for the remaining ones (cvs. Amendoão, Bonita, Casanova, and Pegarinhos-Murça), aldehydes represented the largest fraction of the identified volatiles. Roasting led to an increase in the recorded amount of volatiles, namely in what concerns aldehydes and terpenes, representing the former the major fraction in all cultivars, except for two roasted samples (cvs. Glorieta and Molar). This can indicate that these cultivars are less prone to oxidation, and that they are likely to have better acceptability, due to lower presence of off-flavours caused by the presence of volatile aldehydes. Furthermore, the results obtained clearly indicated an influence of almond cultivar and roasting process in the volatile profile, being these profiles a helpful tool to discriminate cultivars. This was only achieved by the application of chemometric tools (LDA and PCA), which allowed to verify which volatiles were discriminant among varieties and with the application of technological processes.

Acknowledgements Ivo Oliveira is grateful to FCT, POPH-QREN and FSE for the Post-doctoral Fellowship SFRH/BPD/111005/2015. This work is supported by: European Investment Funds by FEDER/ COMPETE/POCI-Operational Competitiveness and Internacionalization Programme, under Project POCI-01-0145-FEDER-006958 and National Funds by FCT-Portuguese Foundation for Science and Technology, under the Project UID/AGR/04033/2013.

\section{Compliance with ethical standards}

Conflict of interest The authors declare that they have no conflict of interest.

\section{References}

Adams R (2007) Identification of essential oil components by gaschromatography/mass spectrometry, 4th edn. Allured Business Media, Illinois

Agila A, Barringer S (2012) Effect of roasting conditions on color and volatile profile including HMF level in sweet almonds (Prunus dulcis). J Food Sci 77:461-468

Beck J, Mahoney N, Cook D, Gee W (2011) Volatile analysis of ground almonds contaminated with naturally occurring fungi. J Agric Food Chem 59:6180-6187

Berenguer-Navarro V, Grané-Teruel N, Martín-Carratalá M (2002) Oxidation stability of almond oil. Acta Hortic 591:125-132

Conn E (1980) Cyanogenic compounds. Annu Rev Plant Biol 31:433-451

Cuevas F, Moreno-Rojas J, Arroyo F, Daza A, Ruiz-Moreno M (2016) Effect of management (organic vs conventional) on volatile profiles of six plum cultivars (Prunus salicina Lindl.). A chemometric approach for varietal classification and determination of potential markers. Food Chem 199:479-484

Dudareva N, Pichersky E, Gershenzon J (2004) Biochemistry of plant volatiles. Plant Physiol 135:1893-1902

Durmaz G, Gökmen V (2010) Determination of 5-hydroxy-methyl-2furfural and 2-furfural in oils as indicators of heat pre-treatment. Food Chem 123:912-916 
Erten E, Cadwallader K (2017) Identification of predominant aroma components of raw, dry roasted and oil roasted almonds. Food Chem 217:244-253

FAOstat (2018) Agriculture Data. http://www.fao.org/faostat/en/ \#data/QC. Accessed 29 Jan 2018

Frankel E (1982) Volatile lipid oxidation products. Prog Lipid Res 22:1-33

Franklin L, Chapman D, King E, Mau M, Huang G, Mitchell A (2017) Chemical and sensory characterization of oxidative changes in roasted almonds undergoing accelerated shelf life. J Agric Food Chem 65:2549-2563

Fugelsang K, Edwards C (2007) Wine microbiology: practical applications and procedures, 2nd edn. Springer, New York

García-Pascual P, Mateos M, Carbonell V, Salazar D (2003) Influence of storage conditions on the quality of shelled and roasted almonds. Biosyst Eng 84:201-209

Kodad O, Alonso J, Espiau Estopañán M, Estopañán G, Juan T (2011) Chemometric characterization of almond germplasm: compositional aspects involved in quality and breeding. J Am Soc Hortic Sci 136:273-281

Krist S, Unterweger H, Bandion F, Buchbauer G (2004) Volatile compound analysis of SPME headspace and extract samples from roasted Italian chestnuts (Castanea sativa Mill.) using GCMS. Eur Food Res Technol 219:470-473

Kwak J, Faranda A, Henkin J, Gallagher M, Preti G, McGovern P (2015) Volatile organic compounds released by enzymatic reactions in raw nonpareil almond kernel. Eur Food Res Technol 241:441-446

Larrauri M, Demaría M, Ryan L, Asensio C, Grosso N, Nepote V (2016) Chemical and sensory quality preservation in coated almonds with the addition of antioxidants. J Food Sci $81: 208-215$

Lee J, Kim D, Chang P (2007) Headspace-solid phase microextraction (HS-SPME) analysis of oxidized volatiles from free fatty acids (FFA) and application for measuring hydrogen donating antioxidant activity. Food Chem 105:414-420

Lee J, Zhang G, Wood E, Rogel-Castillo C, Mitchell A (2013) Quantification of amygdalin in nonbitter, semibitter, and bitter almonds (Prunus dulcis) by UHPLC-(ESI) QqQ MS/MS. J Agric Food Chem 61:7754-7759

Lee J, Xiao L, Zhang G, Ebeler S, Mitchell A (2014) Influence of storage on volatile profiles in roasted almonds (Prunus dulcis). J Agric Food Chem 62:11236-11245

Malheiro R, Pinho P, Soares S, Ferreira A, Baptista P (2013) Volatile biomarkers for wild mushrooms species discrimination. Food Res Int 54:186-194

Malheiro R, Casal S, Rodrigues N, Renard C, Pereira J (2018) Volatile changes in cv. Verdeal Transmontana olive oil: from the drupe to the table, including storage. Food Res Int 106:374-382

McClements D, Decker E (2010) Lípidos. In: Samodaran S, Parkin K, Fennema O, (eds) Fennema quimica de los alimentos. 3th ed. Espana: Editorial Acribia, p 155-214
Mexis S, Badeka A, Kontominas M (2009) Quality evaluation of raw ground almond kernels (Prunus dulcis): effect of active and modified atmosphere packaging, container oxygen barrier and storage conditions. Innov Food Sci Emerg Technol 10:580-589

Min D, Smouse T (1985) Flavor chemistry of fats and oils. American Oil Chemists Society, Urbana, pp 23-31

Oliveira I, Meyer A, Afonso S, Ribeiro C, Gonçalves B (2017) Morphological, mechanical and antioxidant properties of Portuguese almond cultivars. J Food Sci Technol 55:467-478

Oliveira I, Meyer A, Afonso S, Gonçalves B (2018) Compared leaf anatomy and water relations of commercial and traditional Prunus dulcis (Mill.) cultivars under rain-fed conditions. Sci Hortic 229:226-232

Romero A, Vargas F, Tous J, Ninot A, Miarnau X (2011) Industrial aptitude of new almond varieties from IRTA's breeding program. Acta Hortic 912:399-404

Sanahuja A, Santonja M, Teruel N, Carratalá M, Selva M (2011) Classification of almond cultivars using oil volatile compound determination by HS-SPME-GC-MS. J Am Oil Chem Soc 88:329-336

Silva J, Ferreira P (1983) Composição em Ácidos Gordos do Óleo de Algumas Variedades Hortículas da Prunus Dulcis (Miller) DA Wenn da Região do Alto do Douro. Revista da Universidade de Coimbra 29:431-439

Valdés A, Beltrán A, Karabagias I, Badeka A, Kontominas M, Garrigós M (2015) Monitoring the oxidative stability and volatiles in blanched, roasted and fried almonds under normal and accelerated storage conditions by DSC, thermogravimetric analysis and ATR-FTIR. Eur J Lipid Sci Technol 117:1199-1213

Vazquez-Araujo L, Enguix L, Verdu A (2008) Investigation of aromatic compounds in toasted almonds used for the manufacture of turron. Eur Food Res Technol 227:243-254

Whitfield F, Mottram D (1992) Volatiles from interactions of Maillard reactions and lipids. Crit Rev Food Sci Nutr 31:1-58

Xiao L, Lee J, Zhang G, Ebeler S, Wickramasinghe N, Seiber J, Mitchell A (2014) HS-SPME GC/MS characterization of volatiles in raw and dry-roasted almonds (Prunus dulcis). Food Chem 151:31-39

Yada S, Lapsley K, Huang G (2011) A review of composition studies of cultivated almonds: macronutrients and micro-nutrients. J Food Compos Anal 24:469-480

Yang J, Pan Z, Takeoka G, Mackey B, Bingol G, Brandl M, Garcin K, McHugh T, Wang H (2013) Shelflife of infrared dry-roasted almonds. Food Chem 138:671-678

Yildirim A, Akinci-Yildirim F, Şan B, Sesli Y (2016) Total oil content and fatty acid profile of some almond (Amygdalus communis L.) cultivars. Pol J Food Nutr Sci 66:173-178

Publisher's Note Springer Nature remains neutral with regard to jurisdictional claims in published maps and institutional affiliations. 